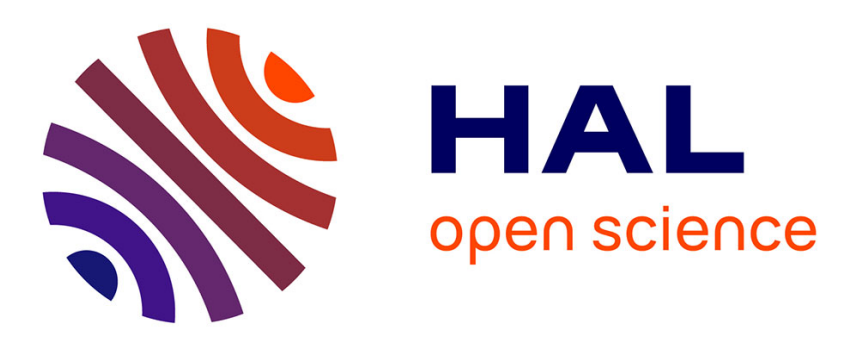

\title{
Arne Naess et l'écologie politique de nos communautés
} Fabrice Flipo

\section{To cite this version:}

Fabrice Flipo. Arne Naess et l'écologie politique de nos communautés. Mouvements : des idées et des luttes, 2009, 60, pp.158-162. 10.3917/mouv.060.0158 . hal-00957949

\section{HAL Id: hal-00957949 \\ https://hal.science/hal-00957949}

Submitted on 11 Mar 2014

HAL is a multi-disciplinary open access archive for the deposit and dissemination of scientific research documents, whether they are published or not. The documents may come from teaching and research institutions in France or abroad, or from public or private research centers.
L'archive ouverte pluridisciplinaire HAL, est destinée au dépôt et à la diffusion de documents scientifiques de niveau recherche, publiés ou non, émanant des établissements d'enseignement et de recherche français ou étrangers, des laboratoires publics ou privés. 
Arne Naess, \{Ecologie, communauté et style de vie\}, Editions MF, 2008, trad. Charles Ruelle.

Par Fabrice Flipo

Arne Naess est l'auteur de la fameuse distinction entre « écologie profonde » et « écologie superficielle » (« deep » et «shallow» ecology[[Voy. Arne Næss, " The Shallow and the Deep, Long-Range Ecology Movement », \{Inquiry\}, n 16, 1973, pp. 95-100.]]), et à ce titre tenu pour être le fondateur de "l'écologie profonde ». L'écologie profonde fait partie, en France, des mouvements qui ont suscité une très forte levée de bouclier. Le pamphlet de Luc Ferry, \{Le Nouvel Ordre Ecologique\}, est l'incarnation la plus médiatique de cette attitude, mais bien d'autres ouvrages ont été publiés pour dénoncer cette pensée, considérée comme absolument infréquentable, malthusienne voire néo-païenne.

L'écologie profonde a été brandie à la manière d'un épouvantail. La question est de savoir pourquoi. Dans les faits, l'écologie profonde a servi à nier l'existence de la crise écologique. La dégradation des écosystèmes s'est accélérée; par exemple, le dernier rapport du GIEC signale que l'accroissement des émissions de gaz à effet de serre a été encore plus rapide que ce qui avait été anticipé dans le plus noir des scénarios construits dans le précédent rapport. Peut-on encore écarter comme prétendument nauséabonde la question des raisons de l'action écologiste ? L'usage moralisant et dénonciateur des textes de Naess doit laisser la place à l'exercice de la pensée critique. Nous ne saurions donc trop remercier les éditions MF pour le service rendu d'avoir rendu accessible au lectorat francophone ce texte important, qui est le premier à être traduit dans notre langue.

Arne Naess est décédé le 14 janvier dernier, à l'âge de 97 ans (1912-2009). Le philosophe norvégien était un spécialiste internationalement reconnu de Spinoza et de Gandhi. En Norvège, son oeuvre a été couronnée de multiples distinctions. Naess a fréquenté le cercle de Vienne. Pacifiste, il s'est engagé dans la résistance contre le nazisme. C'est dire s'il y a loin de la caricature à la réalité. Arne Naess a enseigné à l'université pendant 30 ans avant de se retirer pour s'engager au service de la cause écologiste. Une sélection de ses oeuvres (SWAN) a été publiée chez Springer Verlag.

A la lumière d'un examen attentif, que dit ce texte d'Arne Naess, finalement ? Le souci majeur de l'auteur, dans ce texte qui date pour l'essentiel de 1976, est comprendre le ressort de l'action écologiste, afin d'en favoriser la diffusion rapide. Pourquoi agit-on en faveur des équilibres écologiques ? Quel est le motif d'une telle action? Face aux déséquilibres grandissants, un tel problème devrait mobiliser davantage.

Naess distingue deux réponses face à la crise écologique. La première, la plus courante, s'en prend aux pollutions et à l'épuisement des ressources; elle cherche à " protéger la nature », élaborer des règles de coexistence avec les non-humains etc. Naess estime que cette première approche échoue à infléchir l'évolution du monde : la destruction de la nature continue au même rythme, voire s'accélère. Naess oppose donc une seconde approche : l'écologie " profonde ». La première attitude s'en prend aux effets extérieurs de la crise ; l'écologie profonde se place à sa racine - et cette racine est ontologique. A « l'Homme-dansl'environnement », Naess entend substituer «l'Homme relié avec la nature »: «le problème de la crise environnementale a pour origine le fait que les êtres humains n'ont pas encore pris conscience du potentiel qu'ils ont de vivre des expériences variées \{dans\} et \{de\} la nature » (p. 54, souligné par l'auteur). Tant que l'homme se percevra comme disjoint de la nature, alors il continuera à la détruire sans s'apercevoir qu'il se détruit lui-même. Nous détruisons la nature parce que nous nous vivons mal. Se vivre bien exige de (re)découvrir notre lien avec la nature, ce qui peut être accompli en approfondissant notre Soi. Lorsque nous descendons en nous, au-delà des habitudes et des cadres théoriques hérités, alors nous comprenons qu'être bien implique que la nature soit bien, qu'elle puisse aussi s'épanouir - et nous en elle. Ce n'est qu'à partir de là que nous pouvons trouver des repères pour agir de manière " écologique ». Telle est la démarche de "l'écosophie », en regard de laquelle, estime Naess, toute autre démarche écologiste ne peut être que « superficielle » ("shallow»). L'écologie superficielle s'en prend aux effets externes de la crise car elle ne se rend pas compte que la raison profonde de la crise réside avant tout en nous-mêmes. L'écologie superficielle est donc un discours théorique sans conséquence, sans effet performatif; elle énonce des règles éthiques et annonce le désastre, mais elle ne l'empêche pas de se produire. 

mars 2009 [en ligne]. www.mouvements.info/Arno-Naess-et-l-ecologie-politique.html

Dans un souci pragmatique, Naess a voulu expliciter quelques normes communes aux partisans de l'écologie profonde : c'est la fameuse " plate-forme » en 8 points (\{Voyez sur ce site\} l'article de Baptiste Lanaspeze, [« L'écologie profonde est-elle un humanisme ? »-> art81]), que Naess complète par des normes dérivées, dont il estime qu'elles ne sont que des « fils conducteurs » à ne pas prendre au pied de la lettre. La position défendue se rapproche, Naess le signale lui-même, de l'anarchisme non-violent.

La postface d'H.-S. Afeissa situe les arguments qui ont été opposés, dans la littérature académique, à la position de Naess. Il est bon de les rappeler pour situer le débat. En faisant primer l'ontologie sur l'éthique, Naess aurait fait le lit d'une politique autoritaire. En distinguant «écologie profonde » et "écologie superficielle », Naess aurait inutilement compliqué le débat. La théorie de la "réalisation de Soi » conduirait à abolir l'individu au profit d'un holisme manipulable par de nouveaux gourous. Naess n'aurait pas assez analysé l'origine du rapport d'exploitation à la nature - le capitalisme, le patriarcat. Naess serait resté trop vague au sujet des principes éthiques défendus, oscillant entre écocentrisme (est juste ce qui va dans le sens de l'équilibre de la communauté biotique) et biocentrisme (égalité de principe entre tous les êtres vivants). Le biocentrisme ne mènerait de toute manière qu'à une impasse, car l'égalité de principe est impraticable.

Une partie de ces objections nous semble tenir à une hésitation majeure de l'auteur, rarement prise en compte dans la littérature. Naess dit très clairement se situer dans la perspective d'une sagesse plus que d'un débat éthico-politique (p. 282). Ces sagesses (Lao Tseu, bouddhisme etc.) cherchent à répondre à la question du sens de la vie, elles cherchent à donner à penser et non à rendre compte, abstraire ou formaliser des règles éthiques et morales. Elles préconisent un approfondissement du Soi, de manière à le relier avec le cosmos (Tao etc.), pour arriver à une conscience élargie. On lira notamment à ce sujet l'excellent petit livre d'Eugen Herrigel, \{Le zen dans l'art chevaleresque du tir à l'arc $\}$. Les sagesses se méfient de la connaissance par des concepts, car les concepts figent, nous enferment dans des définitions fixes; Heidegger dirait qu'elles nous enferment dans le « on » impersonnel alors que le plus urgent est de trouver une voie vers l'authenticité. Les sagesses s'appuient sur la pratique, l'intuition, les « exercices spirituels » (Pierre Hadot).

Dès lors le débat entre Naess et ses contradicteurs philosophes rappelle les échanges entre le moine et le philosophe, Matthieu Ricard et son père, Jean-François Revel. Quand le philosophe veut fixer, construire une logique, le moine veut déconstruire, échapper à toute logique formelle, en montrer l'insuffisance radicale pour favoriser un contact intuitif et singulier avec le réel, avec soi. L'un veut formaliser et l'autre veut amener à la prise de conscience, à une transformation du sujet. Pour le moine, formaliser éloigne, enferme, objective, réifie ; pour le philosophe le concept libère. Sur le plan éthique et politique, Naess reprend largement les éthiques environnementales et programmes politiques écologistes existants, sans grande originalité. A vrai dire, ce n'est pas son problème. Ce que veut Naess, c'est amener le lecteur ou la lectrice à se demander si la vie qu'il ou elle a choisie est conforme à une authentique réalisation de soi, en l'amenant en particulier à éprouver les conséquences mortifères de ses choix productivistes. Ensuite, que chacun en tire sa morale pour l'action - et la formalise s'il le souhaite.

Le problème, avec Naess, est qu'il n'a pas su tout à fait choisir entre les deux orientations. Il a développé une sagesse et dans le même temps il a continué de participer au débat académique, par exemple en cherchant à formuler des principes généraux. Cela pose plusieurs problèmes. Le premier est qu'une sagesse destinée à « changer la société » n'est pas la bienvenue en France. La doxa estime que sagesse et politique sont incompatibles, la première relevant strictement de la sphère privée (avec tous les paradoxes de la « laïcité à la française ») et la seconde de la sphère publique. Naess n'aborde pas réellement, dans cet ouvrage, la question de l'articulation entre sagesse et politique. Est-ce lié à un contexte culturel différent ? C'est étonnant car cette problématique est cruciale dans l'approche de Naess, comme le souligne David Rothenberg dans sa préface à l'édition américaine (p. 21). En France le cas de [Pierre Rabhi-> http://www.pierrerabhi.org/blog/index.php], deux fois candidat malheureux à l'élection présidentielle, montre que l'articulation des deux ne va pas de soi.

Second problème, qui est encore une originalité du maître : Naess pose que l'engagement écologiste repose avant sur la défense du bien, et non du juste - à la différence des éthiques environnementales. Or Charles Taylor (\{Les sources $d u$ moi\}, Seuil, 1998) a montré de manière convaincante que les philosophies éthiques et morales occidentales se sont peu à peu repliées sur un procéduralisme fondé en raison (Rawls, Habermas etc.), revendiquant ne prendre aucune part à la définition du bien. Les théories " perfectionnistes », c'est-àdire toutes les théories qui envisagent de définir en quoi consiste une vie humaine réussie, sur le plan 
individuel et collectif, sont soupçonnées de faire le lit du communautarisme, du religieux voire même du totalitarisme. La doxa, à nouveau, veut que le bien, dans nos démocraties de marché, soit donné par le marché, la presse ou l'élection - et non par « la science ». Taylor et Naess récusent cela chacun à leur manière. Ils affirment tous deux que sous le couvert d'un pluralisme proclamé, la discussion sur les conceptions du bien sont, en Occident, maintenues dans d'étroites limites. Pour Naess, le marché et l'Etat se nourrissent tous deux d'une conception de l'Homme en lutte contre la nature, conçue comme avare et hostile, appelant un rapport de force. La nature est " mauvaise » et l'Homme n'est « bon » que lorsqu'il la redresse. La liberté dite «des Modernes» procède ainsi de thèses plus substantielles que ce que ses penseurs ne veulent bien avouer. La raison technique et économique est en effet présentée comme un fait, une norme scientifique, située hors du débat, sur lequel les politiques s'appuient pour produire leurs effets. Mise en cause par la crise écologique, cette raison se dévoile à la fois comme valeur, normativité, au sens d'un comportement souhaitable -et souhaité-, et comme particulière, relative. Bruno Latour confirme cela à sa manière quand il note que l'économie exploite au maximum l'ambiguïté entre les faits et les valeurs, «\{c'est à croire que la Constitution moderniste a été faite pour elle $\} »[[$ Bruno Latour, \{Politiques de la nature\}, La Découverte, 1999, p. 187]]. La croissance économique qui découle de la rationalité moderne n'est pas une simple procédure aboutissant à produire une abondance de moyens qui pourraient être mis au service de toutes les fins possibles, librement poursuivies par les individus jouissant de la plénitude de leurs droits. Le mécanisme social produisant ces moyens, la «mégamachine », est en elle-même une fin; et la poursuite de cette fin particulière annihile d'autres fins possibles, en particulier la pérennité de « la nature » au sens de la « toile de la vie».

Naess s'oppose alors aussi bien aux théories marxiennes classiques, qui défendent le caractère anthropologique du travail et situent l'origine de la crise dans les " rapports de production », qu'aux théories libérales. Chez Naess la centrale nucléaire est un danger pour la nature, qu'elle soit utilisée en mode socialiste ou en mode capitaliste. Il rejoint en cela Marcuse ou Illich. Pour Naess, nous ne vivons pas dans un monde « désenchanté » mais au contraire dans un monde dans lequel « la technologie » et «le marché » exercent toutes sortes de miracles volontairement mis en scène -ainsi la "conquête spatiale ». Dès lors l'objectif avoué de Naess est de nous désaliéner de ce nouveau genre de foi, de nous montrer que ces « miracles » disparaissent dès lors que l'on déshabille la mise en scène et que l'on regarde le tableau dans son ensemble.

Une telle thèse est bien sûr condamnée par les nouveaux prêtres : douter des bienfaits technologiques (OGM, nucléaire, TGV etc.) c'est «s'opposer au Progrès » ou vouloir « revenir à l'âge de pierre ». $\{L a$ nouvelle écologie politique\} de J.-P. Fitoussi, \& E. Laurent (coll. La république des idées, 2008) [[ Fabrice Flipo, «La nouvelle écologie politique - Economie et développement humain », Revue du MAUSS permanente, 17 décembre 2008 [en ligne->http://www.journaldumauss.net/spip.php?article442].]] témoigne bien de cet état de fait : selon les auteurs, être rationnel c'est réduire la rareté par la croissance de la production et avoir confiance dans le progrès technique pour faire face à tous les risques issus de la destruction lente des écosystèmes. Avons-nous le droit de douter des bienfaits technologiques (nanotechnologies, biotechnologies etc.) ? Avons-nous le droit de préférer une forêt au revenu de bois qu'elle pourrait générer? Non, le pluralisme formellement revendiqué s'arrête en pratique à ce qui est conciliable avec l'impératif de croissance, c'est-à-dire avec les « lois » de la mégamachine -voilà qui réduit singulièrement le débat !

Naess n'entend pas pour autant imposer sa conception du bien. Il multiplie les précautions oratoires et va jusqu'à baptiser sa propre démarche d'un nom propre, " écosophie $T$ », afin d'inviter chacun à suivre sa propre voie. Contrairement aux idéologies auxquelles il s'en prend, il ne cherche pas à formuler des « lois de la nature ». Chez Naess en effet la nature n'est pas issue des sciences mais de l'affirmation qu'il est bon d'être dans un monde pourvu d'une diversité luxuriante. Naess n'idéalise pas cette nature, il en admet la rudesse et la cruauté qu'elle peut exhiber; c'est en prenant en compte ces aspects qu'il parvient à ses conclusions. Mais il se méfie aussi, tout comme Bruno Latour, d'une nature qui viendrait " éviscérer» le politique. Naess fait donc un usage plus que mesuré des distinctions issues des scientifiques ; au contraire il cherche constamment à ancrer ses approches dans le sens commun, évitant ainsi d'avaliser la constitution d'une élite détenant «l'écopouvoir», selon le mot de Lascoumes. S'il est bien question d'écologie, c'est d'une écologie du quotidien, opposée à la constitution d'une expertocratie.

Les thèses de Naess sont donc porteuses de profondes remises en cause. Luc Ferry, en un sens, ne s'était pas trompé de cible. Les critiques qu'il adresse indirectement à Naess sont aussi celles qui sont adressées aux 
mouvements écologistes : liberté, démocratie, modernité. Mais les objections ne sont pas constructives. Elles ne relèvent pas le défi écologiste. Elles ne rendent pas compte de leurs propres fondements, qui sont bel et bien mis en cause par la critique écologiste. Pour être rigoureuses, il leur faudrait tout autant répondre aux critiques qui leur son adressées, sans cela l'affrontement n'est qu'un dialogue de sourds. Naess au contraire explique fort bien l'échec historique des solutions purement procédurales telles que les permis ou les taxes, qui ont la faveur des opposants de Naess. La victoire temporaire de Ferry est une victoire à la Pyrrhus car le fait de ne pas se remettre en cause a conduit la doxa à menacer un peu plus les libertés et la démocratie, du fait des risques grandissants. Il ne suffit pas de traiter l'inquiétude des citoyens, comme si les dangers étaient sortis de leur imagination. Naess montre que la crispation sur une définition étroite des libertés a des conséquences majeures. L'interdit pesant sur une partie du débat nécessaire étouffe toute possibilité d'autorégulation; elle conduit au lobbying et à la prolifération réglementaire, à l'image du droit de l'environnement. Chercher à faire de l'écologie sans remettre en cause les lois de la mégamachine "produire plus» et "consommer vert», pour simplifier - génère des régulations contradictoires et hétéronomisantes (p. 203) qui engloutissent peu à peu la démocratie. Pour Ferry et ses partisans, le temps est venu de choisir et de démontrer s'ils sont attachés à la démocratie ou à la protection de leurs privilèges, qui reposent justement sur le pillage d'une nature dérobée au tiers-monde et aux générations à venir.

Comment retrouver la voie d'une autorégulation ? Naess n'est pas très clair. D'un côté il rejoint Rousseau dans l'idée que seules des petites communautés (Gemeinschaft) peuvent être autorégulées de manière démocratique; mais de l'autre il estime ailleurs que des communautés locales peuvent être compatibles avec des systèmes plus centralisés (p. 216). Le lecteur est renvoyé, comme souvent, à son propre contexte et à ses propres choix. Naess n'entend pas devenir une sorte de gourou ou de guide.

Ayant à notre sens fort bien pointé l'objet du débat, Naess nous laisse cependant sur notre faim sur bien des points. La question se pose par exemple de savoir si une sagesse peut faire évoluer une culture et une société, ou si cela reste une voie trop élitiste pour entraîner le changement à grande échelle -c'est un problème qui est posé par " la simplicité volontaire » par exemple. L'exemple de Pierre Rabhi en France témoigne à la fois de cette tentation et de cette difficulté. Par ailleurs, s'il prend position contre l'exploitation, pour l'autogestion, la participation etc. Naess aborde peu la question d'inégalités proprement \{écologiques\}. C'est ici que les critiques écoféministes, anticapitalistes mais aussi réactionnaires portent à plein contre Naess. S'en tenant à la transformation du rapport à la nature, le sage de Tvergastein laisse trop souvent dans l'ombre la diversité des situations humaines et des responsabilités. Or les solutions ne sauraient faire abstraction des conditions initiales à partir desquelles chacun(e) évolue, même d'un point de vue anarchiste et non-violent. 